\title{
Comparison of the Pathological Response and Adverse Effects of Oxaliplatin and Capecitabine versus Paclitaxel and Carboplatin in the Neoadjuvant Chemoradiotherapy Treatment Approach for Esophageal and Gastroesophageal Junction Cancer: A Randomized Control Trial Study
}

\author{
Aida Cheraghi ${ }^{1}$, Maedeh Barahman $^{1}$, Ramyar Hariri $^{2}$, Alireza Nikoofar ${ }^{1} * \mathbb{D}$, Pedram Fadavi $^{3}$ \\ Received: 28 Dec 2020 \\ Published: 25 Oct 2021
}

\begin{abstract}
Background: Neoadjuvant chemoradiation is one of the main treatment approaches in esophageal cancer treatment, which can improve outcomes of a patient with esophageal cancer. In the current study, we aimed to compare the response rate and side effects of 2 distinctive neoadjuvant chemoradiation protocols.

Methods: The study was a randomized clinical trial that was performed on 70 patients with esophageal and gastroesophageal junction cancer in Iran. The study participants were randomly assigned to 1 of our treatment groups. The first group received capecitabine $\left(625 \mathrm{mg} / \mathrm{m}^{2} / \mathrm{TID}\right)$ and oxaliplatin $\left(50 \mathrm{mg} / \mathrm{m}^{2} /\right.$ weekly), while the second group was given a combination of carboplatin (AUC:2/weekly) and paclitaxel $\left(75 \mathrm{mg} / \mathrm{m}^{2} /\right.$ weekly). Both groups were given weekly 50.4-54 Gy dose of RT. Chi square and Fisher exact tests have been used for data analysis. All statistical tests were performed using SPSS software Version 22.0 and the significance level was set at 0.05 .

Results: Complete pathological response was detected in $18(51.4 \%)$ of patients in group I and $8(22.8 \%)$ in group II (p=0.013). We also observed higher thrombocytopenia in CarTax arm 19 (54.2\%) in comparison to CapOX arm 8(2.2.8\%), and the difference was statistically significant $(\mathrm{p}=0.007)$. No statistical difference was found regarding neutropenia, fatigue, anorexia, esophagitis, and diarrhea.
\end{abstract}

Conclusion: The CapOxRT regime provides more favorable outcomes and also it is more tolerated by patients.

Keywords: Neoadjuvant, Chemoradiotherapy, Esophagogastric, Cancer

Conflicts of Interest: None declared

Funding: None

*This work has been published under CC BY-NC-SA 1.0 license.

Copyright $\odot$ Iran University of Medical Sciences

Cite this article as: Cheraghi A, BarahmanM, Hariri R, Nikoofar A, Fadavi P. Comparison of the Pathological Response and Adverse Effects of Oxaliplatin and Capecitabine versus Paclitaxel and Carboplatin in the Neoadjuvant Chemoradiotherapy Treatment Approach for Esophageal and Gastroesophageal Junction Cancer: A Randomized Control Trial Study. Med J Islam Repub Iran. 2021 (25 Oct);35:140. https://doi.org/10.47176/mjiri.35.140

\section{Introduction}

Annually, 480,000 cases of esophageal cancer are diagnosed, making it the sixth leading cause of cancer death and the eighth most common cancer worldwide (1). The

Corresponding author:Dr Alireza Nikoofar, nikoofar@iums.ac.ir

1. Department of Radiotherapy, Firoozgar General Hospital, Iran University of Medical Sciences, Tehran, Iran

2. Obesity Research Center, Research Institute for Endocrine Sciences, Shahid Beheshti University of Medical Sciences, Tehran, Iran

3. Department of Radiotherapy, Shohaday-e-Haftom-e-Tir Hospital, Iran University of Medical Sciences, Tehran, Iran incidence of adenocarcinoma subtype is increasing due to obesity and gastroes ophageal reflux, while the incidence of SCC is declining due to reduced tobacco use and alco-

\section{$\uparrow$ What is "already known" in this topic:}

There are many studies on chemoradiotherapy of esophageal and gastric cancer, most of which have used injectable drugs and less have used oral drugs.

$\rightarrow$ What this article adds:

In this article, unlike the conventional method that uses injectable drugs for esophageal cancer neoadjuvant, we tried to use an oral treatment that is easier to use. 
hol consumption (1).

The 5-year survival rate is low for locally advanced esophageal cancer, although surgery is still the main step in treatment. However, with surgery alone, the 5-year survival rate is between $5 \%$ and $20 \%$ and the mean survival rate is approximately 1 to 2 years (2). Despite the use of modalities, such as MRI, CT scan, and EUS for staging before surgery, $25 \%$ of patients who first have surgery will have a positive microscopic margin (R1), and the 5year survival rate rarely exceeds $40 \%$. Therefore, treatment typically involves several modalities, including radiotherapy, chemotherapy, or chemoradiotherapy before surgery and then surgery $(3,4)$.

For advanced disease, chemotherapy improves survival and quality of life compared with the best supportive care. The regimen containing epirubicin, cisplatin, and infused fluorouracil (ECF) is widely used in Europe $(5,6)$. Capecitabine and oxaliplatin have been also shown as effective as fluorouracil and cisplatin in patients with esophagogastric cancer (7). Preoperative treatment with paclitaxel and carboplatin with chemoradiotherapy is a very tolerable regimen and can be given on an outpatient basis (8).

The CROSS study (9) has provided significant support to the neoadjuvant chemoradiotherapy treatment approach for esophageal cancer, including chemotherapy regimen (weekly carboplatin and paclitaxel for 5 cycles) and radiation therapy conventionally fractionated (41.4 Gy in 1.8 Gy fractions) followed by surgery 4 to 6 weeks after completion of chemoradiotherapy. It has been reported that hematological toxicity (thrombocytopenia) and esophagitis have been the most common side effects of the regimen, including paclitaxel and carboplatin.

In our study, the aim was to evaluate the pathologic response rate with oxaliplatin + capecitabine $+\mathrm{RT}(\mathrm{Ox}-$
CapRT) compared with carboplatin + paclitaxel + RT (CarPacRT) in patients with esophageal and GEJ cancers. The findings of the present study could provide physicians with valuable information for better treatment.

\section{Methods}

\section{Study population}

The study was a randomized clinical trial that was performed on 70 patients with pathologically proven adenocarcinoma or squamous cell carcinoma of the esophagus or gastroesophageal junction (with less than $2 \mathrm{~cm}$ cardia involvement Siewert 1), T2-T4 and/or lymph nodepositive were enrolled in the study. exclusion criteria consisted of Granulocyte count $\leq 1500$, platelet count $\leq 100,000$, creatinine $>120 \mu \mathrm{mol} / \mathrm{L}$, and bilirubin $\geq 1.5$ times the upper limits of normal, FEV $1 \leq 1.2$, no prior history of cancer treatment and severe dysphagia.

Work up procedures, including medical history, physical examination, laboratory assessment, endoscopic ultrasound, and computed tomography (CT) scan of thorax and abdomen with contrast were performed. Patients were randomly divided into 2 equal groups with even and odd numbers; the first group was odd numbers and the second even numbered. The first group (OxCapRT) received oxaliplatin $\left(50 \mathrm{mg} / \mathrm{m}^{2} /\right.$ weekly $)+$ capecitabine $(625$ $\left.\mathrm{mg} / \mathrm{m}^{2} / \mathrm{TID}\right)$ with radiotherapy (50.4-54 Gy $\left.1.8 \mathrm{~Gy} / \mathrm{fr}\right)$ and the second group (CarPacRT) received weekly paclitaxel $\left(75 \mathrm{mg} / \mathrm{m}^{2}\right)+$ carboplatin (AUC:2) with radiotherapy (total dose of 50.4-54 Gy 1.8Gy/fr). The patients underwent surgery 6 to 10 weeks after chemoradiotherapy. All patients were referred to a surgeon (Fig. 1). Pathological specimens were delivered to the pathologist anonymously using even and odd numbers. Adverse effects of the treatments, including hematologic toxicity (neutropenia

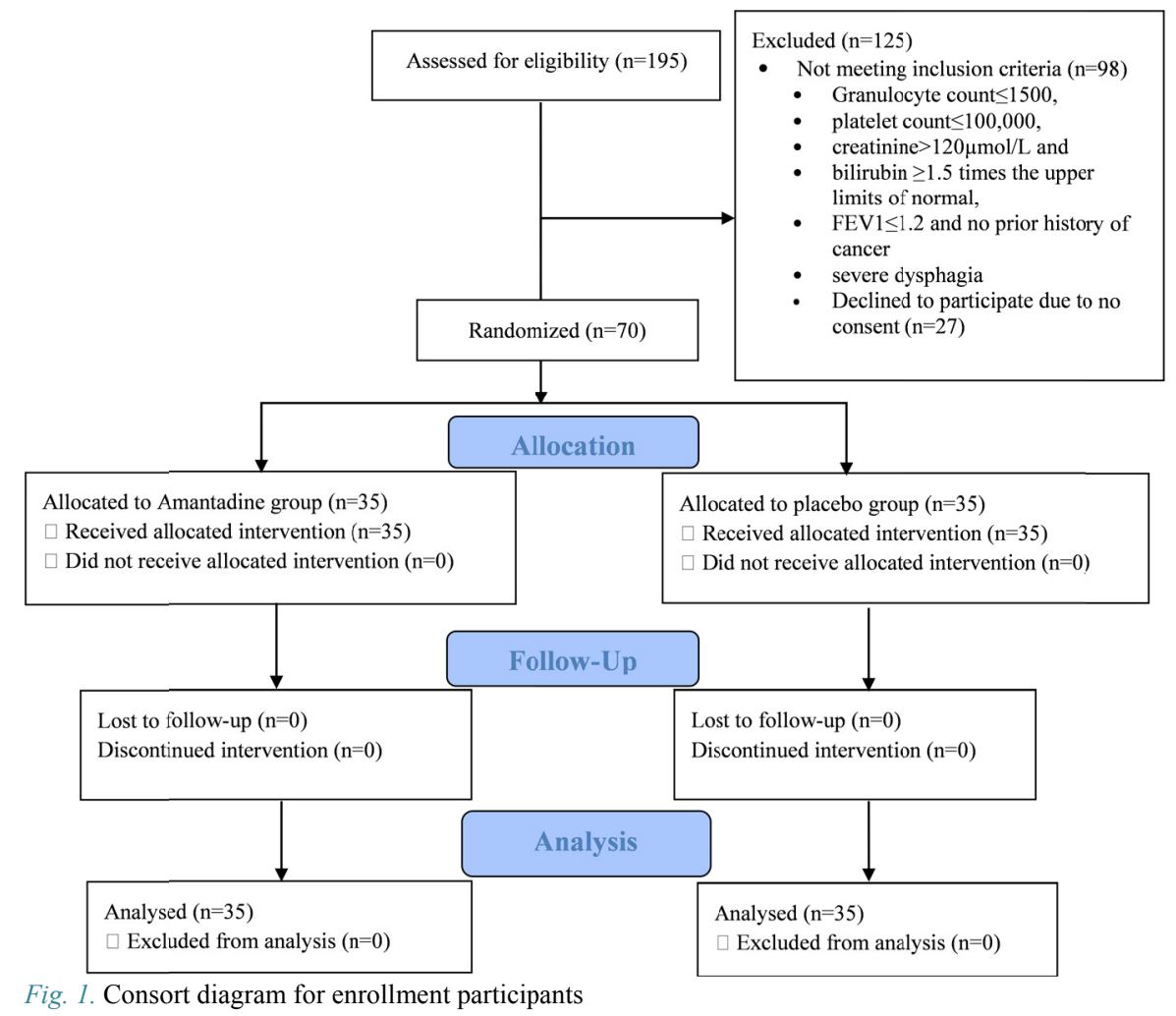


and thrombocytopenia), anorexia, fatigue, diarrhea, and esophagitis, were evaluated during treatment.

This randomized control trial was approved by the Ethics Committee of Iran University of Medical Sciences (approval no, IR.IUMS.FMD.REC.1398.220) and registered by the Iranian Registration of Clinical Trial Center (IRCT) (IRCT20170306032913N4). The study was performed from January 2018 to January 2020 in the department of radiotherapy at Firoozgar hospital affiliated to Iran University of Medical Sciences.

\section{Definitions}

Diarrhea and dysphagia were divided into 3 categories: mild, moderate, and severe. Mild diarrhea was defined as less than 4 times a day, moderate diarrhea 4 to 6 times a day, and severe 7 or more times. Mild dysphagia only has symptoms of difficulty swallowing, moderate dysphagia with difficulty in swallowing solids, and severe dysphagia is difficulty in swallowing liquid and solid. Patients with severe dysphagia were excluded. Esophagitis was also defined according to the Los Angeles criteria (10). Neutropenia was defined as absolute neutrophil count less than 1500 and thrombocytopenia was defined below 100,000 .

Statistical Analysis: Chi square and Fisher exact tests were used to investigate the relationship between each of the qualitative variables and the type of treatment. All statistical tests were performed using SPSS software version 22.0 and the significance level was set at .05.

\section{Results}

According to the results of this study, during 20182020, a total of 70 patients fulfilling admission criteria were prospectively enrolled in the study. Of these, $58(82.9 \%)$ patients had SCC and $12(17.1 \%)$ adenocarcinomas. Also, $16(45.7 \%)$ of the study participants in group I and $17(48.6 \%)$ in group II were women $(p=0.810)$. The mean age $( \pm \mathrm{SD})$ of the participants in groups I and II was reported to be $63.41( \pm 9.7)$ and $61.02( \pm 11.8)$ years, respectively $(\mathrm{p}=0.358)$. Distribution of age, sex, and pathology of study participants and comparison of tumor location, tumor size $(\mathrm{T})$, and lymph node involvement $(\mathrm{N})$ in treatment groups I and II are given in Table 1. The complete pathological response was detected in $18(51.4 \%)$ of patients in group I and $8(22.8 \%)$ in group II ( $\mathrm{p}=0.013)$.

Hematology toxicity, including neutropenia was reported in $9(25.7 \%)$ patients in group I and $10(28.6 \%)$ in group II, and the difference was not significant $(\mathrm{p}=0.788)$. Thrombocytopenia was detected in $8(22.9 \%)$ in group I and $19(54.3 \%)$ in group II ( $\mathrm{p}=0.007)$.

Nonhematologic complications, including esophagitis (mild, moderate, and severe), diarrhea (mild, moderate, and severe), anorexia, and fatigue were not significantly different between the 2 groups. The results are presented in Table 2.

\section{Discussion}

This study was a randomized clinical trial on 70 patients suffering from esophageal and GEJ cancer. Treatment of
Table 1. Distribution of age, sex, and pathology of study participants and Comparison of tumor location, tumor size (T) and lymph node involvement $(\mathrm{N})$ in treatment groups

\begin{tabular}{lccc}
\hline Variable & Group I n (\%) & Group II n (\%) & $\mathrm{p}$ \\
\hline Female, number (\%) & $16(45.7 \%)$ & $17(48.6 \%)$ & 0.810 \\
Age, mean (SD) & $63.41( \pm 9.7)$ & $61.02( \pm 11.8)$ & 0.358 \\
Tumor location & & & \\
$\quad$ Upper & $1(2.8 \%)$ & $2(5.7 \%)$ & 0.555 \\
Middle & $13(37.1 \%)$ & $12(34.2 \%)$ & 0.803 \\
Lower & $21(60.0 \%)$ & $20(57.1 \%)$ & 0.808 \\
T stage & & & \\
T2 & $4(11.4 \%)$ & $2(5.7 \%)$ & 0.393 \\
T3 & $26(74.2 \%)$ & $25(71.4 \%)$ & 0.788 \\
T4 & $5(14.2 \%)$ & $8(22.8 \%)$ & 0.356 \\
N stage & $19(54.2 \%)$ & $12(34.2 \%)$ & 0.092 \\
N1 & $10(28.5 \%)$ & $9(25.7 \%)$ & 0.788 \\
N2 & $5(14.2 \%)$ & $8(22.8 \%)$ & 0.356 \\
N3 & $1(2.8 \%)$ & $6(17.1 \%)$ & 0.046 \\
Unknown & & 0.420 \\
Down stage & $27(77.1 \%)$ & $24(68.5 \%)$ & \\
Yes & $8(22.8 \%)$ & $11(31.4 \%)$ & \\
No & & \\
Morphology & $31(88.6 \%)$ & $27(77.1 \%)$ & \\
SCC, number $(\%)$ & $4(11.4 \%)$ & $8(22.9 \%)$ & \\
ADC, number & & & \\
(\%) & & & \\
\hline T stage, size of tumor; N stage, lymph node involvement; Statistical test: Chi- \\
square and Fisher exact tests.
\end{tabular}

Table 2. Incidence of side effects in treatment groups

\begin{tabular}{lccc}
\hline $\begin{array}{l}\text { Type of complica- } \\
\text { tion }\end{array}$ & $\begin{array}{c}\text { Group I } n \\
(\%)\end{array}$ & $\begin{array}{c}\text { Group II } n \\
(\%)\end{array}$ & $\mathrm{p}$ \\
\hline Neutropenia & $9(25.7 \%)$ & $10(28.6 \%)$ & 0.788 \\
Thrombocytopenia & $8(22.9 \%)$ & $19(54.3 \%)$ & 0.007 \\
Esophagitis & $28(80 \%)$ & $28(80 \%)$ & 1.000 \\
$\quad$ Mild & $8(22.8 \%)$ & $7(20.0 \%)$ & 0.771 \\
$\quad$ Moderate & $15(42.8 \%)$ & $17(48.5 \%)$ & 0.631 \\
$\quad$ Severe & $5(14.2 \%)$ & $4(11.4 \%)$ & 0.721 \\
Diarrhea & $33(94.3 \%)$ & $33(94.3 \%)$ & 1.000 \\
$\quad$ Mild & $25(71.4 \%)$ & $22(62.8 \%)$ & 0.445 \\
$\quad$ Moderate & $5(14.2 \%)$ & $11(31.4 \%)$ & 0.088 \\
$\quad$ Severe & $3(8.6 \%)$ & $0(0 \%)$ & 0.164 \\
Anorexia & $8(22.8 \%)$ & $12(34.2 \%)$ & 0.290 \\
Fatigue & $18(51.4 \%)$ & $18(51.4 \%)$ & 1.000 \\
\hline Statistical test: Chi-square & & &
\end{tabular}

Statistical test: Chi-square and Fisher exact tests.

esophageal cancer has always been a challenge. Chemotherapy, radiotherapy, and surgery are 3 treatments that play an important role in the treatment of esophageal cancer. However, the optimal use of these methods has always been controversial. Few patients with esophageal cancer are resectable and can be treated with a single surgery. However, other patients should be treated with combination therapies that have been proven to improve their outcomes in several clinical trials.

Chemoradiotherapy neoadjuvant is one of the most common therapeutic approaches in the treatment of patients with esophageal cancer and GEJ, the benefits of which have been proven in previous studies. Results from previous studies show that patients undergoing neoadjuvant chemoradiotherapy experience significantly higher survival than those undergoing surgical treatment $(11,12)$. The efficacy of the combination of several chemotherapy drugs, such as platinum, taxanes, and fluoracil, in combination with radiation therapy, in the treatment of patients with esophageal cancer has been demonstrated in several 
studies. Docetaxel and oxaliplatin are other cases that are beneficial in the treatment of patients with esophageal cancer in combination with radiation therapy (13-15). However, there have been few studies comparing different treatment regimens as the optimal drug regimen for neoadjuvant chemoradiotherapy. This study aimed to compare the treatment of capecitabine + oxaliplatin with carboplatin + taxol with radiotherapy for neoadjuvant therapy in patients with esophageal cancer and GEJ. In this study, the pathologic response rate and short-term side effects of both medication regimens were compared.

In a study by Cunningham et al conducted in England, capecitabine and oxaliplatin were evaluated as alternatives to infused fluorouracil and cisplatin, respectively. For untreated advanced esophagogastric cancer, 1002 patients were randomly assigned to receive triplet therapy with epirubicin and cisplatin plus either fluorouracil (ECF) or capecitabine (ECX), or triplet therapy with epirubicin and oxaliplatin plus either fluorouracil (EOF) or capecitabine (EOX). Capecitabine and oxaliplatin were shown to be as effective as fluorouracil and cisplatin, respectively, and oral capecitabine at least as effective as infused fluorouracil and oxaliplatin (which does not require hydration) at least as effective as cisplatin (which does require hydration) for overall survival (7).

Gannett et al evaluated neoadjuvant therapy with weekly paclitaxel/ carboplatin plus 5-fluorouracil (5-FU) with conformal radiotherapy in a phase II trial in 24 patients with T2-4N0-1M0-1a esophageal carcinoma. Patients were treated with paclitaxel $45 \mathrm{mg} / \mathrm{m} 2$ intravenously over 1 hour and carboplatin at an area under the concentrationtime curve (AUC) of 2 intravenously over 30 minutes on days $1,8,15,22$, and 29. Also, 5-Fluorouracil $225 \mathrm{mg} / \mathrm{m} 2$ was administered as continuous infusion on days 1 to 33 . Radiation was delivered to a dose of 45 Gy. Patients underwent surgery 8 weeks after completing chemoradiotherapy. Pathologic complete response occurred in 12 $(57 \%)$ and partial response was reported in 9 patients, including 4 with near complete response. Grade 3 and 4 toxicities included esophagitis in 33\% of patients, hypotension in $29 \%$, stomatitis in $25 \%$, neutropenia in $13 \%$, and anemia in $8 \%$. They concluded that a neoadjuvant chemoradiotherapy by weekly paclitaxel and carboplatin, with 5-FU resulting in high pathological complete remission (pCR) and local control rates and an encouraging 3year survival rate of $48 \%$. Toxicity was substantial but manageable. Further, the distant failure rate was relatively high, which may suggest the need for more active systemic agents (16).

The CROSS study by Hagen et al compared chemoradiotherapy followed by surgery with surgery alone in 368 patients with potentially curable tumors of esophageal or esophagogastric junction cancer. A total of 178 patients received carboplatin (doses titrated to achieve an area under the curve of $2 \mathrm{mg}$ per milliliter per minute) and paclitaxel $\left(50 \mathrm{mg} / \mathrm{m}^{2}\right)$ for 5 weeks and concurrent radiotherapy (41.4 Gy in 23 fractions, 5 days per week), followed by surgery. Also, 188 patients underwent surgery alone. An R0 resection was achieved in 148 of 161 patients $(92 \%)$ in the chemoradiotherapy-surgery group, as compared with 111 of 161 (69\%) in the surgery group (P $<.001)$. Pathological complete response was reported in the resection specimens from 47 patients $(29 \%)$ in the chemoradiotherapy-surgery group. The most frequent hematologic toxic effects observed during chemoradiotherapy were leukopenia (6\%) and neutropenia (2\%). The most frequent nonhematologic toxic effects were anorexia (5\%) and fatigue (3\%). Neoadjuvant chemoradiotherapy improved survival in patients with resectable esophageal or GEJ cancer. The regimen was associated with an acceptable adverse-event rate. In the surgical group, the median survival was improved from 24 to 49.4 months in the modality treatment group. It also caused complete response and $\mathrm{R} 0$ resection in all patients who underwent surgery (17).

A phase 2 study conducted by Pasini et al aimed to define the pathological response of a neoadjuvant regimen, including weekly docetaxel and cisplatin, continuous infusion of 5-fluorouracil (5-FU), and concomitant radiotherapy (RT) in 74 untreated patients with stage 2 and 3 adenocarcinoma and squamous cell carcinoma of the mid-distal thoracic esophagus. Doses were as follows: docetaxel 35 $\mathrm{mg} / \mathrm{m} 2$ and cisplatin $25 \mathrm{mg} / \mathrm{m} 2$ on days $1,8,15,29,36$, 43,50 , and 57 plus $5-\mathrm{FU}(180 \mathrm{mg} / \mathrm{m} 2$ on days $1-21$ and $150 \mathrm{mg} / \mathrm{m} 2$ on days 29-63). RT (50 Gy) started on day 29. Patients underwent surgery 6 to 8 weeks after the completion of chemoradiation. pCR was seen in $47 \%$ of patients and near pCR (microfoci of tumor cells on the primary tumor without lymph nodal metastases) in $15 \%$. Grades 3 and 4 neutropenia occurred in $13.5 \%$, nonhematological toxicity and toxic deaths were reported in $32.4 \%$ and $4 \%$ of the patients, respectively. Their study showed that by using an intensive weekly schedule, a high pathological response was achieved and the subset of patients with pathological complete remission had a statistically higher and durable long-term survival but the side effects of these 3 drugs were high (18).

Our study comparing the efficacy and adverse effects of 2 chemoradiotherapy regimens for the treatment of esophageal and GEJ cancer showed that the alternaltive regimen of capecitabine and oxalliplatin was more efficient, with a higher complete pathologic response than the common regime of paclitaxel and carboplatin. It was also reported that thrombocytopenia was lower in the first group. Other adverse effects were reported similar between the regimens, except for 3 patients with severe diarrhea in group1, suggesting the alternative regimen of weekly capecitabine and oxaliplatin, with concurrent radiotherapy as a promising neoadljuvant treatment for esophageal or GEJ cancers.

One of the main limitations of this study was the coordination problems between the radiotherapy and surgery departments for operating on as soon as possible so that patients can be considered for surgery within the time frame. Another limitation of the study was the impossibility of performing positron emission tomography scans for patients for more accurate staging. Patients with severe dysphagia were not candidates for inclusion in the study, and this was another limitation. Finally, this study needs to be performed with more patients and also a longer fol- 
low-up of patients to assess the survival and recurrence rate.

\section{Conclusion}

The alternative regimen of weekly capecitabine and oxaliplatin with concurrent radiotherapy is more effective with fewer complications compared with the regimen of paclitaxel and carboplatin in the treatment of esophagus or gastroesophageal junction cancers. Our suggestion for future studies is to do it with a larger number of participants.

\section{Acknowledgment}

We would like to thank the staff of the radiotherapy and surgery department of Firoozgar Hospital for their valuable efforts and time.

\section{Conflict of Interests}

The authors declare that they have no competing interests.

\section{References}

1.Zhang Y. Epidemiology of esophageal cancer. World J Gastroenterol. 2013;19(34):5598-606.

2. Allemani C, Matsuda T, Di Carlo V, Harewood R, Matz M, Nikšić M, et al. Global surveillance of trends in cancer survival 2000-14 (CONCORD-3): analysis of individual records for 37513025 patients diagnosed with one of 18 cancers from 322 population-based registries in 71 countries. Lancet (London, England). 2018;391(10125):1023-75.

3. Kim TJ, Kim HY, Lee KW, Kim MS. Multimodality assessment of esophageal cancer: preoperative staging and monitoring of response to therapy. Radiographics. 2009;29(2):403-21.

4. Napier KJ, Scheerer M, Misra S. Esophageal cancer: A Review of epidemiology, pathogenesis, staging workup and treatment modalities. World J Gastrointest Oncol. 2014;6(5):112-20.

5. Ross P, Nicolson M, Cunningham D, Valle J, Seymour M, Harper P, et al. Prospective randomized trial comparing mitomycin, cisplatin, and protracted venous-infusion fluorouracil (PVI 5-FU) With epirubicin, cisplatin, and PVI 5-FU in advanced esophagogastric cancer. J Clin Oncol. 2002;20(8):1996-2004.

6. Webb A, Cunningham D, Scarffe JH, Harper P, Norman A, Joffe JK, et al. Randomized trial comparing epirubicin, cisplatin, and fluorouracil versus fluorouracil, doxorubicin, and methotrexate in advanced esophagogastric cancer. J Clin Oncol. 1997;15(1):261-7.

7. Cunningham D, Okines AF, Ashley S. Capecitabine and oxaliplatin for advanced esophagogastric cancer. N Eng J Med. 2010;362(9):8589.

8. van Meerten E, Muller K, Tilanus HW, Siersema PD, Eijkenboom WM, van Dekken $\mathrm{H}$, et al. Neoadjuvant concurrent chemoradiation with weekly paclitaxel and carboplatin for patients with oesophageal cancer: a phase II study. Br J Cancer. 2006;94(10):1389-94.

9. Pepek JM WC, Czito BG. The CROSS trial: End of the debate on neoadjuvant therapy for esophageal cancer? Int $\mathrm{J}$ Clin Pract. 2012;9(6):607-9.

10. Lundell LR, Dent J, Bennett JR, Blum AL, Armstrong D, Galmiche JP, et al. Endoscopic assessment of oesophagitis: clinical and functional correlates and further validation of the Los Angeles classification. Gut J. 1999;45(2):172-80.

11. Urba SG, Orringer MB, Turrisi A, Iannettoni M, Forastiere A, Strawderman M. Randomized trial of preoperative chemoradiation versus surgery alone in patients with locoregional esophageal carcinoma. J Clin Oncol. 2001;19(2):305-13.

12. Walsh TN, Noonan N, Hollywood D, Kelly A, Keeling N, Hennessy TP. A comparison of multimodal therapy and surgery for esophageal adenocarcinoma. N Eng J Med. 1996;335(7):462-7.

13. Al-Batran SE, Hofheinz RD, Pauligk C, Kopp HG, Haag GM, Luley $\mathrm{KB}$, et al. Histopathological regression after neoadjuvant docetaxel, oxaliplatin, fluorouracil, and leucovorin versus epirubicin, cisplatin, and fluorouracil or capecitabine in patients with resectable gastric or gastro-oesophageal junction adenocarcinoma (FLOT4-AIO): results from the phase 2 part of a multicentre, open-label, randomised phase 2/3 trial. Lancet Oncol. 2016;17(12): :1697-708.

14. Al-Batran SE, Homann N, Pauligk C, Goetze TO, Meiler J, Kasper $\mathrm{S}$, et al. Perioperative chemotherapy' with fluorouracil plus leucovorin, oxaliplatin, and docetaxel versus fluorouracil or capecitabine plus cisplatin and epirubicin for locally advanced, resectable gastric or gastro-oesophageal junction adenocarcinoma (FLOT4): a randomised, phase 2/3 trial. Lancet (London, England). 2019;393(10184):1948-57.

15. Cartwright E, Cunningham D. The Role of Systemic Therapy in Resectable Gastric and Gastro-oesophageal Junction Cancer. Curr Treat Opt Oncol J. 2017;18(12):69.

16. Gannett DE, Wolf RF, Takahashi GW, Louie J, Wagner RC, Ey FS, et al. Neoadjuvant chemoradiotherapy for esophageal cancer using weekly Paclitaxel and Carboplatin plus infusional 5-Fluorouracil. Gastrointest Cancer Res Offic J. 2007;1(4):132-8.

17. van Hagen $\mathrm{P}$, Hulshof MC, van Lanschot JJ, Steyerberg EW, van Berge Henegouwen MI, Wijnhoven BP, et al. Preoperative chemoradiotherapy for esophageal or junctional cancer N Eng J Med. 2012;366(22):2074-84.

18. Pasini F, de Manzoni G, Zanoni A, Grandinetti A, Capirci C, Pavarana M, et al. Neoadjuvant therapy with weekly docetaxel and cisplatin, 5-fluorouracil continuous infusion, and concurrent radiotherapy in patients with locally advanced esophageal cancer produced a high percentage of long-lasting pathological complete response: a phase 2 study. Cancer. 2013;119(5):939-45. 